International Journal of Pure and Applied Mathematics

Volume 96 No. 2 2014, 289-298

ISSN: 1311-8080 (printed version); ISSN: 1314-3395 (on-line version)

url: http://www.ijpam.eu

doi: http://dx.doi.org/10.12732/ijpam.v96i2.10

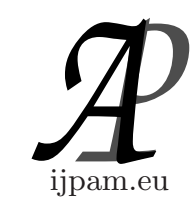

\title{
A NOTE ON SYMMETRIC $k$-TRIDIAGONAL MATRIX FAMILY AND THE FIBONACCI NUMBERS
}

\author{
Fatih Ylmaz ${ }^{1}$, Tomohiro Sogabe ${ }^{2}$ \\ ${ }^{1}$ Department of Mathematics \\ Polatlı Art and Science Faculty \\ Gazi University \\ 06900, Ankara, TURKEY \\ ${ }^{2}$ Graduate School of Information Science \\ Technology Aichi Prefectural University \\ Aichi 480-1198, JAPAN
}

\begin{abstract}
In this note, we provide arbitrary integer powers for another type of $k$-tridiagonal matrix family whose integer powers are specified to the famous Fibonacci numbers.
\end{abstract}

AMS Subject Classification: 15A18, 15A15

Key Words: $k$-tridiagonal matrix, Chebyshev polynomial, determinant, Fibonacci numbers

\section{Introduction}

The $k$-tridiagonal matrix [8] has received much attenson in recent years in the field of special matrices. $T_{n}^{(k)}$ is defined as follows:

Received: July 14, 2014

(C) 2014 Academic Publications, Ltd.

$\S$ Correspondence author url: www.acadpubl.eu 


$$
T_{n}^{(k)}=\left(\begin{array}{cccccccc}
d_{1} & 0 & \cdots & 0 & a_{1} & 0 & \cdots & 0 \\
0 & d_{2} & & & & a_{2} & & \vdots \\
\vdots & & \ddots & & & & \ddots & 0 \\
0 & & & d_{n-k} & & & & a_{n-k} \\
b_{k+1} & & & & \ddots & & & 0 \\
0 & b_{k+2} & & & & \ddots & & \vdots \\
\vdots & & \ddots & & & & d_{n-1} & 0 \\
0 & \ldots & 0 & b_{n} & 0 & \ldots & 0 & d_{n}
\end{array}\right)
$$

It has recently been found that [9] the $k$-tridiagonal matrix plays important role in describing generalized $k$-Fibonacci numbers. Moreover, the authors ([3]-[6]) computed integer powers of some special types of these matrices by exploiting some properties of Chebyshev polynomials. The authors ([8]-[10]) investigated $k$-tridiagonal matrices. The paper ([13]) shows the importance of powers of a matrix for a generalized Fibonacci numbers, where elements of the matrix powers generates the generalized Fibonacci numbers. This also motivates us to find such matrices for ordinary Fibonacci numbers.

Among numerical sequences, the Fibonacci numbers which is defined by the recurrence $F_{n+2}=F_{n+1}+F_{n}$ for $n \geq 0$, where $F_{0}=0$ and $F_{1}=1$, has achieved a kind of celebrity status [2]. Binet defined an explicit formula for Fibonacci numbers [2] using the roots of quadratic equation $x^{2}-x-1=0$ as

$$
F_{n}=\frac{\alpha^{n}-\beta^{n}}{\alpha-\beta},
$$

where $\alpha$ is positive root and $\beta$ is negative root of the equation. It is well known that the determinant of a special tridiagonal matrix yields Fibonacci numbers, see, e.g., [1] for the recent overview.

In this note, we consider a specialized form of symmetric $k$-tridiagonal matrix, i.e.,

$$
A_{n, k}= \begin{cases}1, & a_{i, i+k}=a_{i+k, i}, \text { for } i=1,2, \ldots, 3 k, \\ 0, & \text { otherwise. }\end{cases}
$$

where $n=4 k(k=1,2, \ldots)$. The matrix (2) is not only theoretical interest, but arises in scientific computing, e.g. a finite difference discretization of Laplace operator over thin rectangular domain.

The aim of this note is to compute integer powers of these type of matrices and show that all integer powers of $A$ are specified to the famous Fibonacci numbers with positive and negative powers. 


\section{Eigenvalues of $A_{n, k}$}

Lemma 1 ([4]). Let $\triangle_{n}(\alpha)$ be of the form

$$
\triangle_{n}(\alpha)=\operatorname{det}\left(\begin{array}{ccccc}
\alpha & 1 & & & \\
1 & \alpha & 1 & & \\
& \ddots & \ddots & \ddots & \\
& & 1 & \alpha & 1 \\
& & & 1 & \alpha
\end{array}\right)
$$

Then it holds

$$
\triangle_{n}(\alpha)=\alpha \triangle_{n-1}(\alpha)-\triangle_{n-2}(\alpha)
$$

with $\triangle_{0}(\alpha)=1, \triangle_{1}(\alpha)=\alpha, \triangle_{2}(\alpha)=\alpha^{2}-1$. Moreover, the solution of difference equation (3) is $\triangle_{n}(\alpha)=U_{n}(\alpha / 2)$. Here $U_{n}(\alpha / 2)$ is the $n$th Chebyshev polynomial of the second kind:

$$
U_{n}(x)=\frac{\sin (n+1) \arccos x}{\sin \arccos x}, \quad-1 \leq x \leq 1 .
$$

All roots of $U_{n}(x)$ are included in the interval $[-1,1]$ and can be found using the relation

$$
x=\cos \frac{k \pi}{n+1}, \quad k=1,2, \ldots, n .
$$

Proposition 2. All eigenvalues of $A_{n, k}$ are of the form

$$
\lambda_{k}=-2 \cos \frac{k \pi}{5}, \quad k=1,2,3,4 .
$$

Proof. Let $P_{n}(x)=\left|A_{n, k}-x I\right|$ be the characteristic polynomial of the matrix $A_{n, k}$. Then, $P_{n}(x)=\left[\triangle_{4}(-x)\right]^{\frac{n}{4}}$. Since $n=4 k$, it follows from Lemma 1 that

$$
P_{n}(x)=\left[U_{4}\left(-\frac{x}{2}\right)\right]^{k} .
$$

Thus, from Lemma 1 , zeros of $P_{n}(x)$ are given by $\lambda_{k}=-2 \cos (k \pi / 5), k=$ $1,2,3,4$. This completes the proof.

Proposition 2 may be interesting since all eigenvalues of $A_{n, k}$ does not depend on matrix size $n$. Moreover, we can write

$$
\begin{aligned}
P_{n}(x) & =\left(x^{4}-3 x^{2}+1\right)^{\frac{n}{4}} \\
& =\left[\left(x^{2}-1+x\right)\left(x^{2}-1-x\right)\right]^{\frac{n}{4}}
\end{aligned}
$$




$$
=\left[\left(x^{2}-\alpha^{2}\right)\left(x^{2}-\beta^{2}\right)\right]^{\frac{n}{4}},
$$

where $\alpha=(1+\sqrt{5}) / 2$ and $\beta=(1-\sqrt{5}) / 2$. All eigenvalues of the matrix are multiple and $\left(l_{k}=k, k=1,2, \ldots, n / 4\right)$ is the multiplicity of the eigenvalue of $\lambda_{k}$. Then, we write Jordan's form of the matrix $A_{n, k}$ as:

$$
J=\operatorname{diag}\left(\lambda_{1}, \ldots, \lambda_{1}, \lambda_{2}, \ldots, \lambda_{2}, \lambda_{3}, \ldots, \lambda_{3}, \lambda_{4}, \ldots, \lambda_{4}\right)
$$

From (5) and Proposition 2, we easily have the following result:

Corollary 3. Let $A$ be $n$-square matrix as in (2). Then

$$
\operatorname{det}\left(A_{n, k}\right)=1
$$

Proof. From Proposition 2, the product of all eigenvalues is

$$
\prod_{i=1}^{4}\left(-2 \cos \frac{i \pi}{5}\right)^{k}=\left[\prod_{i=1}^{4}\left(-2 \cos \frac{i \pi}{5}\right)\right]^{k}=1^{k}=1 .
$$

This concludes the proof.

\section{Eigenvectors of $A_{n, k}$ and Transforming Matrix}

Proposition 4. Let $T$ be a matrix such that $J=T^{-1} A_{n, k} T$, where matrix $J$ corresponds to $(5)$. Let $m_{i}=\left(4-\lambda_{i}^{2}\right) /(2 n+2)$. Let $T_{i}$ 's and $t_{i}$ 's are submatrices of $T$ and $T^{-1}$ such that $T=\left(T_{1}, T_{2}, T_{3}, T_{4}\right)$ and $T^{-1}=\left(t_{1}, t_{2}, t_{3}, t_{4}\right)$ respectively. Then, $T_{i}$ 's and $t_{i}$ 's are given by 


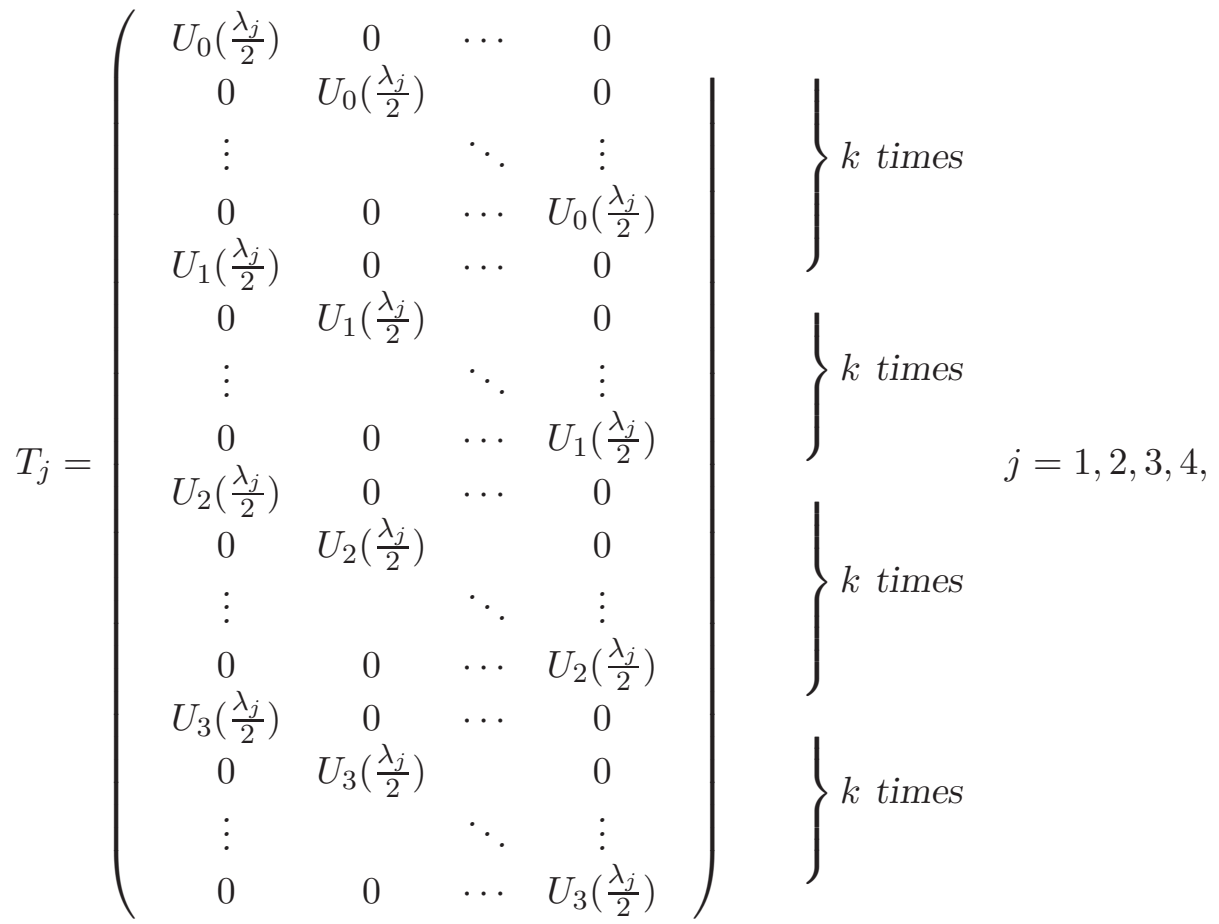

$$
\begin{aligned}
& \left.t_{j}=\left(\begin{array}{cccc}
m_{1} U_{j-1}\left(\frac{\lambda_{1}}{2}\right) & 0 & \cdots & 0 \\
0 & m_{1} U_{j-1}\left(\frac{\lambda_{1}}{2}\right) & & 0 \\
\vdots & 0 & \cdots & m_{1} U_{j-1}\left(\frac{\lambda_{1}}{2}\right) \\
0 & 0 & \cdots & 0 \\
m_{2} U_{j-1}\left(\frac{\lambda_{2}}{2}\right) & m_{2} U_{j-1}\left(\frac{\lambda_{2}}{2}\right) & & 0 \\
0 & & \ddots & \vdots \\
\vdots & 0 & \cdots & m_{2} U_{j-1}\left(\frac{\lambda_{2}}{2}\right) \\
0 & 0 & \cdots & 0 \\
m_{3} U_{j-1}\left(\frac{\lambda_{3}}{2}\right) & m_{3} U_{j-1}\left(\frac{\lambda_{3}}{2}\right) & & 0 \\
0 & & \ddots & \vdots \\
\vdots & 0 & \cdots & m_{3} U_{j-1}\left(\frac{\lambda_{3}}{2}\right) \\
0 & 0 & \cdots & 0 \\
m_{4} U_{j-1}\left(\frac{\lambda_{4}}{2}\right) & m_{4} U_{j-1}\left(\frac{\lambda_{4}}{2}\right) & & 0 \\
0 & & \ddots & \vdots \\
\vdots & 0 & \cdots & m_{4} U_{j-1}\left(\frac{\lambda_{4}}{2}\right)
\end{array}\right\}\right\} k \text { times }
\end{aligned}
$$

where $j=1,2,3,4$. 
We now give the main result.

Theorem 5. Let $A_{n, k}$ be a matrix as in (2). Then

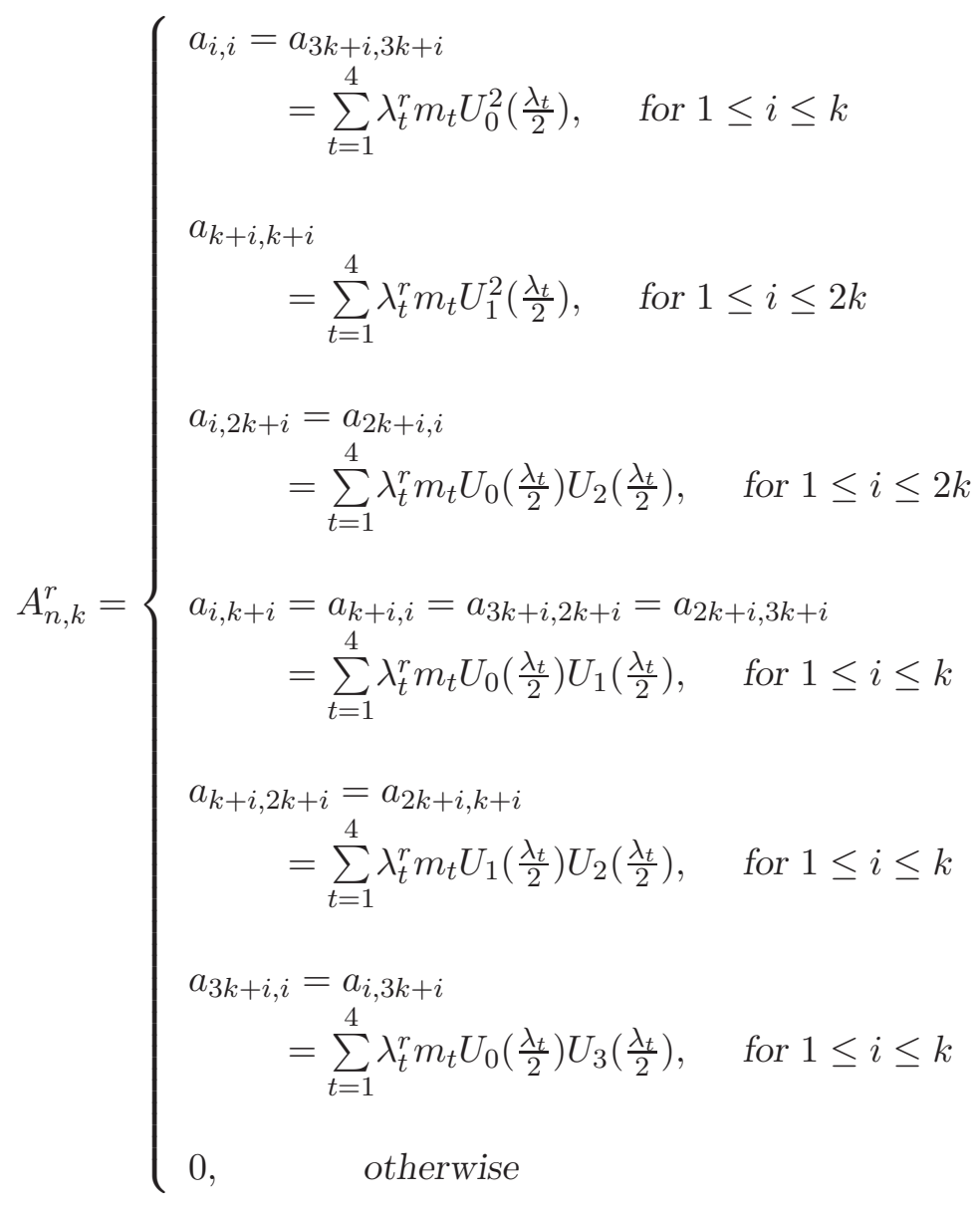

here $r$ is any arbitrary integer.

Proof. By matrix multiplication (5) and Proposition 4, we obtain the expression which is desired.

Moreover it can be seen that arbitrary integer power of the matrix can be given as below: 


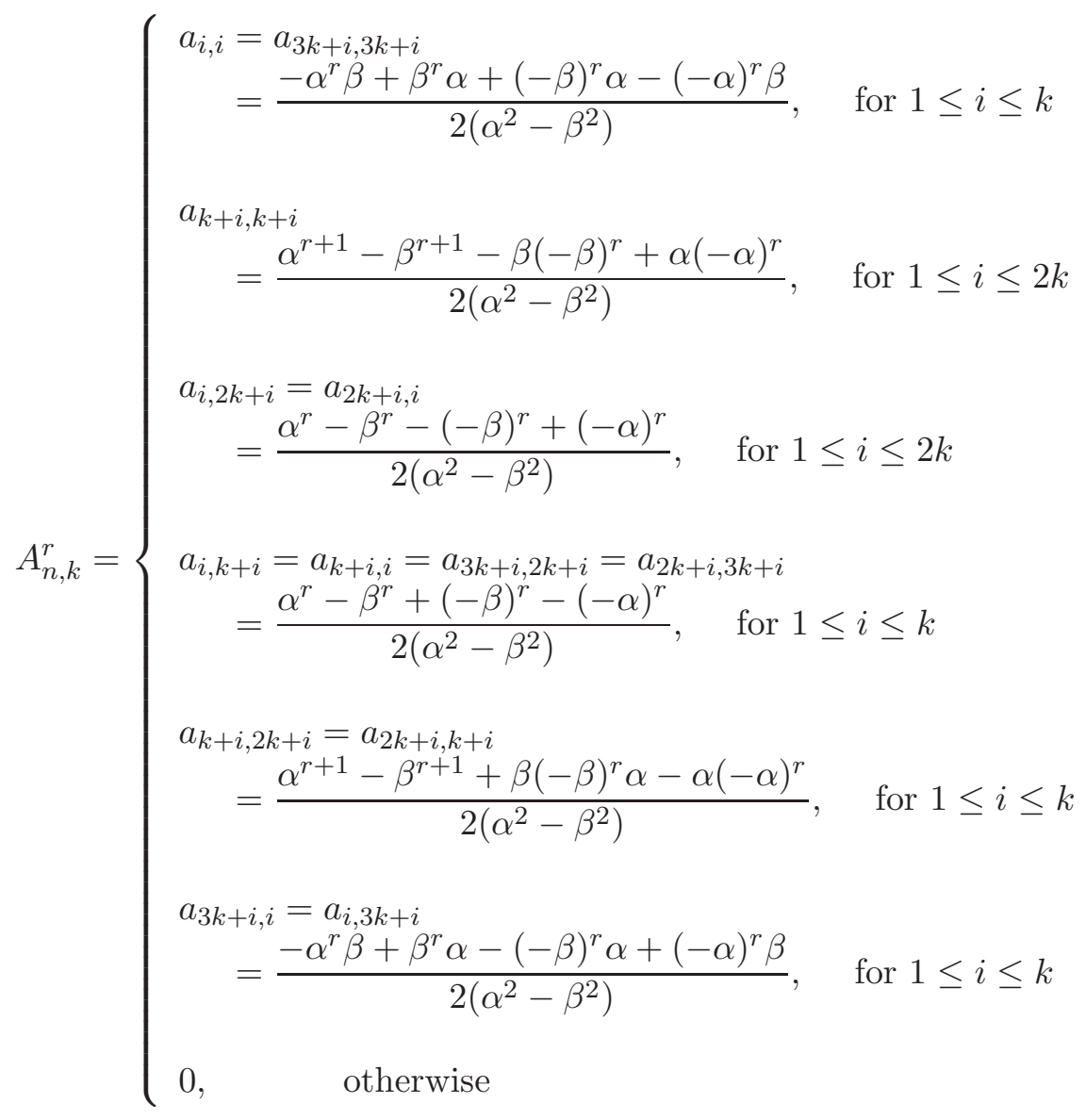

here $\alpha=\frac{1+\sqrt{5}}{2}$ and $\beta=\frac{1-\sqrt{5}}{2}$. 


\section{Illustrative Examples}

We can find arbitrary integer powers of the matrix $A_{n, k}$, taking into account derived expressions. For example, let $k=2$, then $n=8$, i.e.,

$$
A_{8,2}=\left(\begin{array}{cccccccc}
0 & 0 & 1 & 0 & 0 & 0 & 0 & 0 \\
0 & 0 & 0 & 1 & 0 & 0 & 0 & 0 \\
1 & 0 & 0 & 0 & 1 & 0 & 0 & 0 \\
0 & 1 & 0 & 0 & 0 & 1 & 0 & 0 \\
0 & 0 & 1 & 0 & 0 & 0 & 1 & 0 \\
0 & 0 & 0 & 1 & 0 & 0 & 0 & 1 \\
0 & 0 & 0 & 0 & 1 & 0 & 0 & 0 \\
0 & 0 & 0 & 0 & 0 & 1 & 0 & 0
\end{array}\right) .
$$

The transforming matrix of $A_{8,2}$ is

$$
T=\left(\begin{array}{cccccccc}
U_{0}\left(\frac{\lambda_{1}}{2}\right) & 0 & U_{0}\left(\frac{\lambda_{2}}{2}\right) & 0 & U_{0}\left(\frac{\lambda_{3}}{2}\right) & 0 & U_{0}\left(\frac{\lambda_{4}}{2}\right) & 0 \\
0 & U_{0}\left(\frac{\lambda_{1}}{2}\right) & 0 & U_{0}\left(\frac{\lambda_{2}}{2}\right) & 0 & U_{0}\left(\frac{\lambda_{3}}{2}\right) & 0 & U_{0}\left(\frac{\lambda_{4}}{2}\right) \\
U_{1}\left(\frac{\lambda_{1}}{2}\right) & 0 & U_{1}\left(\frac{\lambda_{2}}{2}\right) & 0 & U_{1}\left(\frac{\lambda_{3}}{2}\right) & 0 & U_{1}\left(\frac{\lambda_{4}}{2}\right) & 0 \\
0 & U_{1}\left(\frac{\lambda_{1}}{2}\right) & 0 & U_{1}\left(\frac{\lambda_{2}}{2}\right) & 0 & U_{1}\left(\frac{\lambda_{3}}{2}\right) & 0 & U_{1}\left(\frac{\lambda_{4}}{2}\right) \\
U_{2}\left(\frac{\lambda_{1}}{2}\right) & 0 & U_{2}\left(\frac{\lambda_{2}}{2}\right) & 0 & U_{2}\left(\frac{\lambda_{3}}{2}\right) & 0 & U_{2}\left(\frac{\lambda_{4}}{2}\right) & 0 \\
0 & U_{2}\left(\frac{\lambda_{1}}{2}\right) & 0 & U_{2}\left(\frac{\lambda_{2}}{2}\right) & 0 & U_{2}\left(\frac{\lambda_{3}}{2}\right) & 0 & U_{2}\left(\frac{\lambda_{4}}{2}\right) \\
U_{3}\left(\frac{\lambda_{1}}{2}\right) & 0 & U_{3}\left(\frac{\lambda_{2}}{2}\right) & 0 & U_{3}\left(\frac{\lambda_{3}}{2}\right) & 0 & U_{3}\left(\frac{\lambda_{4}}{2}\right) & 0 \\
0 & U_{3}\left(\frac{\lambda_{1}}{2}\right) & 0 & U_{3}\left(\frac{\lambda_{2}}{2}\right) & 0 & U_{3}\left(\frac{\lambda_{3}}{2}\right) & 0 & U_{3}\left(\frac{\lambda_{4}}{2}\right)
\end{array}\right) .
$$

The inverse of the matrix $T$ is

$$
\begin{aligned}
& T^{-1}=\operatorname{diag}\left(m_{1}, m_{1}, m_{2}, m_{2}, m_{3}, m_{3}, m_{4}, m_{4}\right) \cdot \\
& \left.\qquad \begin{array}{cccccccc}
U_{0}\left(\frac{\lambda_{1}}{2}\right) & 0 & U_{1}\left(\frac{\lambda_{1}}{2}\right) & 0 & U_{2}\left(\frac{\lambda_{1}}{2}\right) & 0 & U_{3}\left(\frac{\lambda_{1}}{2}\right) & 0 \\
0 & U_{0}\left(\frac{\lambda_{1}}{2}\right) & 0 & U_{1}\left(\frac{\lambda_{1}}{2}\right) & 0 & U_{2}\left(\frac{\lambda_{1}}{2}\right) & 0 & U_{3}\left(\frac{\lambda_{1}}{2}\right) \\
U_{0}\left(\frac{\lambda_{2}}{2}\right) & 0 & U_{1}\left(\frac{\lambda_{2}}{2}\right) & 0 & U_{2}\left(\frac{\lambda_{2}}{2}\right) & 0 & U_{3}\left(\frac{\lambda_{2}}{2}\right) & 0 \\
0 & U_{0}\left(\frac{\lambda_{2}}{2}\right) & 0 & U_{1}\left(\frac{\lambda_{2}}{2}\right) & 0 & U_{2}\left(\frac{\lambda_{2}}{2}\right) & 0 & U_{3}\left(\frac{\lambda_{2}}{2}\right) \\
U_{0}\left(\frac{\lambda_{3}}{2}\right) & 0 & U_{1}\left(\frac{\lambda_{3}}{2}\right) & 0 & U_{2}\left(\frac{\lambda_{3}}{2}\right) & 0 & U_{3}\left(\frac{\lambda_{3}}{2}\right) & 0 \\
0 & U_{0}\left(\frac{\lambda_{3}}{2}\right) & 0 & U_{1}\left(\frac{\lambda_{3}}{2}\right) & 0 & U_{2}\left(\frac{\lambda_{3}}{2}\right) & 0 & U_{3}\left(\frac{\lambda_{3}}{2}\right) \\
U_{0}\left(\frac{\lambda_{4}}{2}\right) & 0 & U_{1}\left(\frac{\lambda_{4}}{2}\right) & 0 & U_{2}\left(\frac{\lambda_{4}}{2}\right) & 0 & U_{3}\left(\frac{\lambda_{4}}{2}\right) & 0 \\
0 & U_{0}\left(\frac{\lambda_{4}}{2}\right) & 0 & U_{1}\left(\frac{\lambda_{4}}{2}\right) & 0 & U_{2}\left(\frac{\lambda_{4}}{2}\right) & 0 & U_{3}\left(\frac{\lambda_{4}}{2}\right)
\end{array}\right),
\end{aligned}
$$

and Jordan form is

$$
J=\operatorname{diag}\left(\lambda_{1}, \lambda_{1}, \lambda_{2}, \lambda_{2}, \lambda_{3}, \lambda_{3}, \lambda_{4}, \lambda_{4}\right) .
$$


For $r=4,5$,

$$
A_{8,2}^{4}=\left(\begin{array}{cccccccc}
2 & 0 & 0 & 0 & 3 & 0 & 0 & 0 \\
0 & 2 & 0 & 0 & 0 & 3 & 0 & 0 \\
0 & 0 & 5 & 0 & 0 & 0 & 3 & 0 \\
0 & 0 & 0 & 5 & 0 & 0 & 0 & 3 \\
3 & 0 & 0 & 0 & 5 & 0 & 0 & 0 \\
0 & 3 & 0 & 0 & 0 & 5 & 0 & 0 \\
0 & 0 & 3 & 0 & 0 & 0 & 2 & 0 \\
0 & 0 & 0 & 3 & 0 & 0 & 0 & 2
\end{array}\right), \quad A_{8,2}^{5}=\left(\begin{array}{llllllll}
0 & 0 & 5 & 0 & 0 & 0 & 3 & 0 \\
0 & 0 & 0 & 5 & 0 & 0 & 0 & 3 \\
5 & 0 & 0 & 0 & 8 & 0 & 0 & 0 \\
0 & 5 & 0 & 0 & 0 & 8 & 0 & 0 \\
0 & 0 & 8 & 0 & 0 & 0 & 5 & 0 \\
0 & 0 & 0 & 8 & 0 & 0 & 0 & 5 \\
3 & 0 & 0 & 0 & 5 & 0 & 0 & 0 \\
0 & 3 & 0 & 0 & 0 & 5 & 0 & 0
\end{array}\right) .
$$

For $r=-4$,

$$
A_{8,2}^{-4}=\left(\begin{array}{cccccccc}
5 & 0 & 0 & 0 & -3 & 0 & 0 & 0 \\
0 & 5 & 0 & 0 & 0 & -3 & 0 & 0 \\
0 & 0 & 2 & 0 & 0 & 0 & -3 & 0 \\
0 & 0 & 0 & 2 & 0 & 0 & 0 & -3 \\
-3 & 0 & 0 & 0 & 2 & 0 & 0 & 0 \\
0 & -3 & 0 & 0 & 0 & 2 & 0 & 0 \\
0 & 0 & -3 & 0 & 0 & 0 & 5 & 0 \\
0 & 0 & 0 & -3 & 0 & 0 & 0 & 5
\end{array}\right)
$$

For $r=-5$,

$$
A_{8,2}^{-5}=\left(\begin{array}{cccccccc}
0 & 0 & 5 & 0 & 0 & 0 & -8 & 0 \\
0 & 0 & 0 & 5 & 0 & 0 & 0 & -8 \\
5 & 0 & 0 & 0 & -3 & 0 & 0 & 0 \\
0 & 5 & 0 & 0 & 0 & -3 & 0 & 0 \\
0 & 0 & -3 & 0 & 0 & 0 & 5 & 0 \\
0 & 0 & 0 & -3 & 0 & 0 & 0 & 5 \\
-8 & 0 & 0 & 0 & 5 & 0 & 0 & 0 \\
0 & -8 & 0 & 0 & 0 & 5 & 0 & 0
\end{array}\right)
$$

From these examples, we see that all nonzero elements in the powers of matrix $A_{n, k}$ are closely related to Fibonacci numbers.

\section{References}

[1] J. Jina and P. Trojovsky, On determinants of some tridiagonal matrices connected with Fibonacci numbers, Int. J. Pure and Appl. Math., 88 (2013), 569-575. 
[2] T. Koshy, Fibonacci and Lucas Numbers with Applications, WileyInterscience Publication, 2001.

[3] J. Rimas, On computing of arbitrary positive integer powers for one type of symmetric tridiagonal matrices of even order-I, Appl. Math. Comput., 168 (2005) 783-787.

[4] J. Rimas, On computing of arbitrary positive integer powers for one type of symetric pentadiagonal matrices of even order, Appl. Math. Comput., 203 (2008) 582-591.

[5] J. Rimas, On computing of arbitrary positive integer powers for one type of symmetric pentadiagonal matrices of odd order, Appl. Math. Comput., 204 (2008) 120-129.

[6] J. Rimas, On computing of arbitrary positive integer powers for one type of symmetric tridiagonal matrices of even order II, Appl. Math. Comput., 172 (2006) 245-251.

[7] H. Lütkepohl, Handbook of Matrices, John Wiley-Sons Ltd., 1996, England.

[8] T. Sogabe, M. El-Mikkawy, Fast block diagonalization of $k$-tridiagonal matrices, Appl. Math. Comput., 218 (2011) 2741-2743.

[9] M.E.A. El-Mikkawy, T. Sogabe, A new family of $k$-Fibonacci numbers, Appl. Math. Comput., 215 (2010) 4456-4461.

[10] J. Jia, T. Sogabe, M. El-Mikkawy, Inversion of $k$-tridiagonal matrices with Toeplitz structure, Comput. Math. Appl., 65 (2013) 116-125.

[11] C. M. da Fonseca, On the eigenvalues of some tridiagonal matrices, J. Comput. Appl. Math., 200 (2007) 283-286.

[12] M. S. Solary, Finding eigenvalues for heptadiagonal symmetric Toeplitz matrices, J. Math. Anal. Appl., 402 (2013) 719-730.

[13] E. Kilic, The Binet formula, sums and representations of generalized Fibonacci p-numbers, European J. Combin., 29 (2008) 701-711. 